\title{
HOXB9 promotes endometrial cancer progression by targeting E2F3
}

\author{
Junhu Wan', Hongyang Liư, Quanling Feng ${ }^{2}$, Jun Liư ${ }^{3}$ and Liang Ming ${ }^{1}$
}

\begin{abstract}
HOXB9, as a HOX family transcription factor, playing a significant role in embryonic development and cancer progression. However, the function of HOXB9 and its precise mechanism in regulating endometrial cancer progression remains unknown. Here, we demonstrated that the expression of HOXB9 was increased in endometrial cancer, and associated with histological grade and lymph node metastasis. In addition, elevated HOXB9 predicts a poor prognosis in endometrial cancer patients. Interestingly, bioinformatics analysis of TCGA cancer database showed that HOXB9 expression is positively correlated with E2F3 expression. Moreover, HOXB9 promoted E2F3 expression by directly targeting to its promoter. Furthermore, we found that knocking down E2F3 abolished the ability of HOXB9 in enhancing cell migration. Taken together, for the first, we demonstrated the function and mechanism of HOXB9 in regulating endometrial cancer progression, and indicated HOXB9 may be a novel prognostic marker of endometrial cancer.
\end{abstract}

\section{Introduction}

Endometrial cancer (EC) is one of the leading causes of gynecologic malignancies ${ }^{1,2}$. The patients present with high-grade tumors are aggressive, and frequently diagnosed with tumors spread beyond the uterus ${ }^{3,4}$. Surgery is the major treatment for EC, while it is quite important to develop new therapeutic strategies for $\mathrm{EC}^{5}$. The investigation of new molecular mechanisms in EC may be useful to identify new diagnostic and therapeutic targets.

The HOX genes, encode a group of transcription factors, share a highly conserved homeobox domain. In vertebrates, the whole $39 \mathrm{HOX}$ genes are identified and grouped into four clusters (HOXA, B, C and D) ${ }^{6-8}$. During embryogenesis, sequential HOX expression from $3^{\prime}$ to $5^{\prime}$ along the anteriorposterior (AP) axis could control

\footnotetext{
Correspondence: Junhu Wan (wanjh2009@163.com) or

Liang Ming (mingliang3703@163.com)

'Department of Clinical Laboratory, The First Affiliated Hospital of Zhengzhou University, 450052 Zhengzhou, Henan, China

${ }^{2}$ Department of Obstetrics and Gynecology, The Third Affiliated Hospital of Zhengzhou University, 450052 Zhengzhou, Henan, China

Full list of author information is available at the end of the article

These authors contributed equally: Junhu Wan, Hongyang Liu.

Edited by G.M. Fimia.
}

body segmentation according to the rules of spatial and temporal collinearity ${ }^{8-11}$. In particular, HOXB9 together with other HOX genes controls the specification of thoracic skeletal elements and mammary gland development ${ }^{12,13}$.

In addition to its key roles in embryo development, HOXB9 was also found to be participated in the regulation of numerous human cancers ${ }^{14-17}$. In the previous study, we found HOXB9-regulated lung adenocarcinoma progression by directly targeting JMJD $6^{18}$. In addition, upregulation of HOXB9 showed poor overall survival in invasive human breast cancer, and promoted epithelialto-mesenchymal transition $(\mathrm{EMT})^{19-21}$. However, increased expression of HOXB9 showed better overall survival in colon adenocarcinoma, pancreatic ductal adenocarcinoma and gastric carcinoma patients ${ }^{15-17}$, indicating HOXB9 played an opposite role in these cancer progression.

The E2F family of transcription factors is conserved from nematodes to mammals, which were identified as factors that control the cell cycle, differentiation, apoptosis and stress responses ${ }^{22,23}$. As an important member 
of E2F family, E2F3, encodes two isoforms, E2f3a and E2f3b, whose expression is driven by distinct promoters ${ }^{24}$. Overexpressed E2F3 expression has been reported in ovarian, prostate and lung cancers ${ }^{25-27}$. In the thyroid cancer mouse model, a role for E2F3 has been found in the metastasis of tumor cells to the liver and lung ${ }^{28}$. Using conditional knockout approaches, Trikha et al. reported that ablation of E2F3 in tumor-associated macrophages (TAMs) results in decreased pulmonary metastasis without affecting the tumor growth ${ }^{29}$. Tsuruta et al. reported that the expression of E2F3 was upregulated in $28.6 \%$ (20 of 70) of EC cases $^{30}$. However, the role of E2F3 in EC progression remains unclear.

Although HOXB9 has been extensively investigated in some cancer types, its role in EC has never been reported. In this report, we demonstrated that HOXB9 is overexpressed in EC, and is correlated with EC cell migration. Moreover, HOXB9 promotes EC progression by targeting oncogenic protein E2F3.

\section{Results}

The expression of HOXB9 and its clinical significance in EC

To determine the expression of HOXB9 in EC, we analyzed its expression in a series of 88 endometrial carcinoma, 15 normal proliferative endometrium and 21 atypical endometrial hyperplasia by immunohistochemistry. The expression of HOXB9 was observed mainly in the nucleus of cells. The staining scores of HOXB9 expression level were dichotomized into two groups, low (score of 0 or 1 ) and high (score of 2 or 3). The representative images of "low" and "high" staining, as well as negative controls, in endometrial carcinoma, normal proliferative endometrium and atypical hyperplasia tissues were shown in Fig. 1a. We found that the high expression ratio and expression level of HOXB9 in normal proliferative endometrium, atypical endometrial hyperplasia and endometrial carcinoma were gradually increased $(P=0.0196$; Fig. $1 \mathrm{~b}$ and S1a). The correlations between HOXB9 expression and clinicopathologic characteristics of endometrial carcinoma are shown in Table 1. In endometrial carcinoma, HOXB9 expression correlated only with histological grade $(P=0.0081)$ and lymph node metastasis status $(P=0.001$; Fig. 1c). The HOXB9 expression score in G1, G2 and G3 was gradually increased (Fig. S1b) and tumors with metastasis expressed significantly higher levels than tumors without metastasis (Fig. S1c). However, no correlation between HOXB9 expression with patients' age, surgical staging and muscular invasion endomembrane (Table 1). In addition, we found high HOXB9 expression level correlated significantly with shorter disease-specific survival time as illustrated in Fig. 1d.

Besides, the results of TCGA database, which were mainly analyzed by the web-based tools, Gene Expression
Profiling Interactive Analysis (GEPIA, http://gepia.cancerpku.cn/) $)^{31}$ and the LinkedOmics database (http://www. linkedomics.org) ${ }^{32}$, showed that HOXB9 expression was significantly overexpressed in human EC tissues compared with normal tissues (Fig. 1e and Table 2), and elevated HOXB9 expression level corresponds to a shorter overall survival in EC (Fig. 1f). These findings suggested that HOXB9 is highly expressed in EC and elevated HOXB9 may predict a poor outcome for EC patients.

\section{HOXB9 promotes EC cell migration}

Given the high expression level of HOXB9 in EC, we first investigated the effect of HOXB9 on cancer cell proliferation. However, when endogenous HOXB9 in EC cell line Ishikawa was knocked down with two different HOXB9 small interfering RNA (siRNA), there was no difference in the cell proliferation rate in the three groups (Fig. 2a). Then, we performed colony formation assays using the Ishikawa stable cell lines infected with lentiviruses carrying control short hairpin RNA (shRNA) or HOXB9 shRNA. However, the colony formation assays also showed that the depletion of HOXB9 in Ishikawa cells resulted in no significant decrease in the colony number (Fig. 2b). Moreover, mouse xenograft assays using the Ishikawa stable cell lines infected with lentiviruses carrying control shRNA or HOXB9 shRNA showed that cells expressing sh-HOXB9 displayed no significance in tumor growth rate and tumor volumes than that of the control group (Figs. 2c-e). It indicated that HOXB9 did not influence the proliferation ability of EC cell.

We then tested the EC cell migration mediated by HOXB9. Using transwell assays, we found that cell migration ability was decreased when knocked down HOXB9 in Ishikawa and RL95-2 cells (Figs. 2f, g). In contrast, after overexpression of HOXB9 in the two cancer cells, the cell migration ability markedly increased (Figs. 2h, i). To extend the study of the precise functional role of HOXB9 changed in vivo in regulating EC progression, we introduced a doxycycline (Dox)-inducible system, which allows the induction of HOXB9 by the addition of Dox in EC cell line Ishikawa. Western blot analysis revealed the expression of HOXB9 was markedly increased by adding Dox (Fig. 2j). Using transwell assays, we demonstrated that Dox-induced HOXB9 expression significantly increased cell migration capacity compared with the controls \pm Dox or HOXB9-inducible cells without Dox induction (Figs. 2k, l). In addition, we performed wound-healing assays to determine whether HOXB9 affects the cancer cell migration. Similarly to the previous report, knocked down HOXB9 inhibited Ishikawa cell migration, and overexpression of HOXB9 accelerated migration of Ishikawa cell (Figs. 2m, n). Thus, these data suggested that HOXB9 mainly affect the EC cell migration, not cell proliferation. 


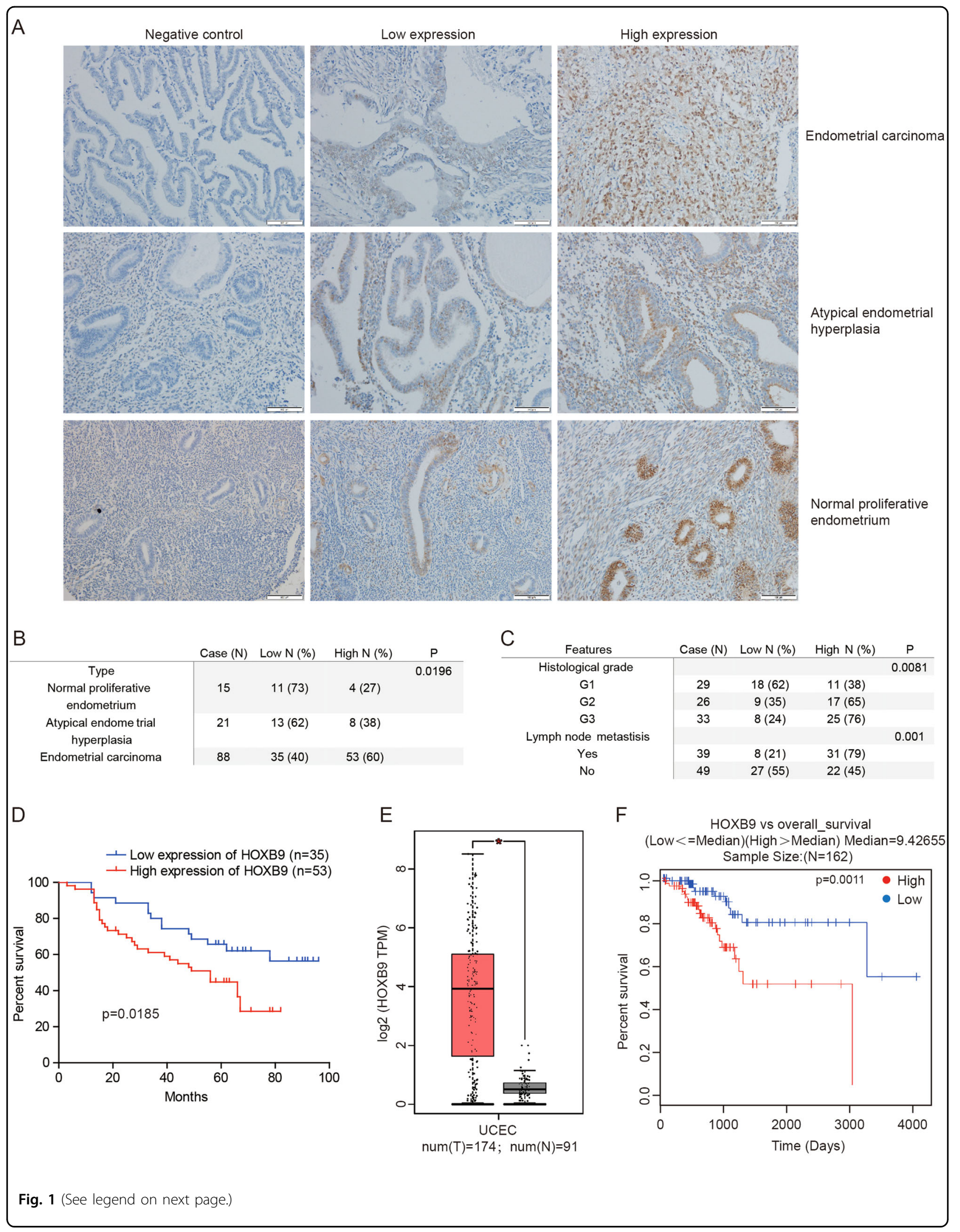


(see figure on previous page)

Fig. 1 The expression of НОХВ9 and its clinical significance in endometrial cancer. a The staining scores of HOXB9 expression level were dichotomized into two groups, low (score of 0 or 1) and high (score of 2 or 3). We have displayed the representative images of "low" and "high" staining, as well as negative controls, in endometrial carcinoma, normal proliferative endometrium and atypical hyperplasia tissues. $\mathbf{b}$ The high expression ratio of HOXB9 in normal proliferative endometrium, atypical endometrial hyperplasia and endometrial carcinoma were showed. c The HOXB9 expression correlation in endometrial carcinoma with histological grade and lymph node metastasis status. $\mathbf{d}$ The Kaplan-Meier analysis of HOXB9 expression in endometrial carcinoma was performed. e, $\mathbf{f}$ The expression level of HOXB9 in human endometrial cancer compared with normal tissues in TCGA database $\mathbf{e}$, and the Kaplan-Meier analysis of HOXB9 expression in human endometrial cancer in TCGA $\mathbf{f}$. The statistical analyses were performed by one-way ANOVA analyses $\mathbf{b}$, c , Student's $t$-test $\mathbf{e}$ and Kaplan-Meier analysis d, f. ${ }^{*} P<0.05$

Table 1 Relationship between HOXB9 expression and clinicopathological features in endometrial carcinomas

\begin{tabular}{|c|c|c|c|c|}
\hline \multirow[b]{2}{*}{ Features } & \multicolumn{4}{|l|}{ HOXB9 } \\
\hline & Case $(N)$ & $\begin{array}{l}\text { Low } \\
N(\%)\end{array}$ & $\begin{array}{l}\text { High } \\
N(\%)\end{array}$ & $P$-value \\
\hline Age & & & & 0.2396 \\
\hline$\leq 50$ & 41 & $19(46)$ & $22(54)$ & \\
\hline$>50$ & 47 & $16(34)$ & $31(66)$ & \\
\hline Surgical staging & & & & 0.1041 \\
\hline I & 53 & $19(36)$ & $34(64)$ & \\
\hline$\|$ & 18 & $11(61)$ & $7(39)$ & \\
\hline III-IV & 17 & $5(29)$ & $12(71)$ & \\
\hline Histological grade & & & & 0.0081 \\
\hline G1 & 29 & $18(62)$ & $11(38)$ & \\
\hline $\mathrm{G} 2$ & 26 & $9(35)$ & $17(65)$ & \\
\hline G3 & 33 & $8(24)$ & $25(76)$ & \\
\hline $\begin{array}{l}\text { Muscular invasion } \\
\text { endomembrane }\end{array}$ & & & & 0.2446 \\
\hline$\leq 1 / 2$ & 39 & $12(31)$ & $27(69)$ & \\
\hline$>1 / 2$ & 49 & $21(43)$ & $28(57)$ & \\
\hline Lymph node metastisis & & & & 0.001 \\
\hline Yes & 39 & $8(21)$ & $31(79)$ & \\
\hline No & 49 & $27(55)$ & $22(45)$ & \\
\hline
\end{tabular}

\section{The expression between HOXB9 and E2F3 is positively correlated}

To investigate the signal pathways regulated by HOXB9 in EC, we analyzed series of genes that have strong coexpression correlation (Pearson $r$ value $>0.2$ or $<-0.2$ ) with HOXB9 in TCGA from the LinkedOmics database (http://www.linkedomics.org) (Figs. 3a, b). Next, we performed Gene ontology (GO) and Kyoto Encyclopedia of Genes and Genomes (KEGG) enrichment analysis on the correlated expressed genes. The enrichment analysis of GO biological process revealed that these genes were involved in a variety of processes, including P53, p38 Mitogen-activated Protein Kinase (MAPK), and Ataxia
Telangiectasia Mutated Kinase (ATM) and integrin family cell surface interaction pathways (Fig. 3c). Meanwhile, KEGG enrichment analysis demonstrated that these genes were closely related to multiple pathways, including transport, regulation of cell cycle, metabolism and energy pathways (Fig. 3d).

Among the total positively or negatively correlated significant genes, E2F3 was noted to act as oncogenic protein $^{33-35}$. E2F3 was reported overexpressed in multiple tumors, and correlated with cancer metastasis. When we examined the mRNA expression levels of several tumor metastasis-related genes after knocking down HOXB9 (data not shown), we only found E2F3 mRNA expression levels were decreased significantly (Fig. 4c). Then, we analyzed the correlation of HOXB9 and E2F3 expression in TCGA EC database and found a positive correlation between HOXB9 and E2F3 mRNA expression levels $(R=$ 0.33, $P=8.5 \mathrm{e}-06$; Fig. 3e). In addition, the mRNA expression level of E2F3 was higher in EC than the normal tissues (Fig. S1d), and the high expression level of E2F3 correlated with the poor outcome of EC patients (Fig. S1e). To further clarify the expression of E2F3 in EC, we analyzed its expression in a series of 88 endometrial carcinoma using immunohistochemical staining. The staining scores of E2F3 expression level were dichotomized into two groups, low and high (Fig. S1f). Interestingly, we also found high E2F3 expression level correlated significantly with shorter survival time ( $P=0.003$; Fig. $3 f)$. Meanwhile, we analyzed the correlation of HOXB9 and E2F3 expression in 88 endometrial carcinoma. Consistent with the result in TCGA database, we found the expression levels of HOXB9 was correlated with E2F3 expression $(P=0.0252$; Fig. $3 g)$. These results indicated that E2F3 indeed acts as an oncogenic protein in EC.

\section{E2F3 is a direct downstream target of HOXB9}

Interestingly, we discovered that knocked down HOXB9 by two different siRNA in Ishikawa cells inhibited the protein expression of E2F3, and overexpression of HOXB9 increased protein levels of E2F3 (Figs. 4a, b). In addition, E2F3 mRNA expression levels were decreased when knocked down HOXB9 (Fig. 4c), indicating that E2F3 may be a transcriptional target of HOXB9. To 
Table 2 The sample information on TCGA database for endometrial carcinoma

\begin{tabular}{|c|c|c|c|c|}
\hline Sample ID & Sample_type & Clinical_stage & -OS & Histological_type \\
\hline TCGA-AX-A05S-01 & Primary tumor & Stage IIIC & 413 & Endometrioid endometrial adenocarcinoma \\
\hline TCGA-AP-A05P-01 & Primary tumor & Stage IA & 636 & Endometrioid endometrial adenocarcinoma \\
\hline TCGA-AP-A05O-01 & Primary tumor & Stage IB & 384 & Endometrioid endometrial adenocarcinoma \\
\hline TCGA-AP-A05N-01 & Primary tumor & Stage IA & 726 & Endometrioid endometrial adenocarcinoma \\
\hline TCGA-DI-A2QU-11 & Primary tumor & Stage IVB & 946 & Endometrioid endometrial adenocarcinoma \\
\hline TCGA-AJ-A2QN-01 & Primary tumor & Stage II & 1069 & Endometrioid endometrial adenocarcinoma \\
\hline TCGA-AJ-A2QL-11 & Primary tumor & Stage IIIC1 & 602 & Endometrioid endometrial adenocarcinoma \\
\hline TCGA-AJ-A2QK-01 & Primary tumor & Stage IA & 643 & Endometrioid endometrial adenocarcinoma \\
\hline TCGA-EY-A2OM-01 & Primary tumor & Stage IA & 694 & Endometrioid endometrial adenocarcinoma \\
\hline TCGA-BG-A2L7-11 & Primary tumor & Stage IA & 612 & Endometrioid endometrial adenocarcinoma \\
\hline TCGA-DF-A2KS-01 & Primary tumor & Stage IB & 3318 & Endometrioid endometrial adenocarcinoma \\
\hline TCGA-AX-A2HK-01 & Primary tumor & Stage IIIC & 90 & Endometrioid endometrial adenocarcinoma \\
\hline TCGA-AX-A2HJ-01 & Primary tumor & Stage IC & 935 & Endometrioid endometrial adenocarcinoma \\
\hline TCGA-AX-A2HG-01 & Primary tumor & Stage IC & 1163 & Endometrioid endometrial adenocarcinoma \\
\hline TCGA-AX-A2HD-01 & Primary tumor & Stage IIIA & 1414 & Endometrioid endometrial adenocarcinoma \\
\hline TCGA-AX-A2HC-01 & Primary tumor & Stage IIIC & 1044 & Endometrioid endometrial adenocarcinoma \\
\hline TCGA-AX-A2HA-11 & Primary tumor & Stage IB & 2475 & Endometrioid endometrial adenocarcinoma \\
\hline TCGA-AX-A2H8-11 & Primary tumor & Stage IB & 1861 & Endometrioid endometrial adenocarcinoma \\
\hline TCGA-AX-A2H7-11 & Primary tumor & Stage IC & 1241 & Endometrioid endometrial adenocarcinoma \\
\hline TCGA-D1-A2G6-01 & Primary tumor & Stage IIIC & 258 & Endometrioid endometrial adenocarcinoma \\
\hline TCGA-D1-A2G5-01 & Primary tumor & Stage IB & 1455 & Endometrioid endometrial adenocarcinoma \\
\hline TCGA-FI-A2F9-01 & Primary tumor & Stage IA & 1803 & Endometrioid endometrial adenocarcinoma \\
\hline TCGA-FI-A2F4-01 & Primary tumor & Stage IC & 2230 & Endometrioid endometrial adenocarcinoma \\
\hline TCGA-FI-A2D6-01 & Primary tumor & Stage IB & 767 & Endometrioid endometrial adenocarcinoma \\
\hline TCGA-FI-A2D5-01 & Primary tumor & Stage IVB & 421 & Endometrioid endometrial adenocarcinoma \\
\hline TCGA-FI-A2D4-01 & Primary tumor & Stage IIIC & 243 & Endometrioid endometrial adenocarcinoma \\
\hline TCGA-FI-A2D0-01 & Primary tumor & Stage IA & 1023 & Endometrioid endometrial adenocarcinoma \\
\hline TCGA-FI-A2CX-01 & Primary tumor & Stage IB & 2031 & Endometrioid endometrial adenocarcinoma \\
\hline TCGA-BG-A2AE-11 & Primary tumor & Stage IB & 721 & Endometrioid endometrial adenocarcinoma \\
\hline TCGA-BG-A2AD-01 & Primary tumor & Stage $\| \mathrm{B}$ & 609 & Endometrioid endometrial adenocarcinoma \\
\hline TCGA-EC-A1QX-01 & Primary tumor & Stage IB & 224 & Endometrioid endometrial adenocarcinoma \\
\hline TCGA-AW-A1PO-01 & Primary tumor & Stage IIIA & 17 & Endometrioid endometrial adenocarcinoma \\
\hline TCGA-A5-A1OK-01 & Primary tumor & Stage IIIA & 917 & Endometrioid endometrial adenocarcinoma \\
\hline TCGA-A5-A1OJ-01 & Primary tumor & Stage IA & 1006 & Endometrioid endometrial adenocarcinoma \\
\hline TCGA-D1-A1O8-01 & Primary tumor & Stage IIIC & 120 & Endometrioid endometrial adenocarcinoma \\
\hline TCGA-D1-A1O7-01 & Primary tumor & Stage IA & 32 & Endometrioid endometrial adenocarcinoma \\
\hline TCGA-D1-A1O5-01 & Primary tumor & Stage IA & 458 & Endometrioid endometrial adenocarcinoma \\
\hline TCGA-D1-A1O0-01 & Primary tumor & Stage IA & 63 & Endometrioid endometrial adenocarcinoma \\
\hline TCGA-D1-A1NZ-01 & Primary tumor & Stage IA & 548 & Endometrioid endometrial adenocarcinoma \\
\hline TCGA-D1-A1NY-01 & Primary tumor & Stage IB & 513 & Endometrioid endometrial adenocarcinoma \\
\hline
\end{tabular}


Table 2 continued

\begin{tabular}{|c|c|c|c|c|}
\hline Sample ID & Sample_type & Clinical_stage & _os & Histological_type \\
\hline TCGA-D1-A1NS-01 & Primary tumor & Stage IA & 43 & Endometrioid endometrial adenocarcinoma \\
\hline TCGA-DI-A1NO-11 & Primary tumor & Stage IIIA & 285 & Endometrioid endometrial adenocarcinoma \\
\hline TCGA-EC-A1NJ-01 & Primary tumor & Stage IA & 488 & Endometrioid endometrial adenocarcinoma \\
\hline TCGA-B5-A1MZ-01 & Primary tumor & Stage IA & 1598 & Endometrioid endometrial adenocarcinoma \\
\hline TCGA-B5-A1MX-01 & Primary tumor & Stage IB & 1473 & Endometrioid endometrial adenocarcinoma \\
\hline TCGA-B5-A1MV-01 & Primary tumor & Stage IA & 1565 & Endometrioid endometrial adenocarcinoma \\
\hline TCGA-B5-A1MR-01 & Primary tumor & Stage IIIA & 6859 & Endometrioid endometrial adenocarcinoma \\
\hline TCGA-E6-A1M0-11 & Primary tumor & Stage IIIC & 1793 & Endometrioid endometrial adenocarcinoma \\
\hline TCGA-E6-A1LX-01 & Primary tumor & Stage IB & 711 & Endometrioid endometrial adenocarcinoma \\
\hline TCGA-EY-A1H0-01 & Primary tumor & Stage $I I I C 2$ & 588 & Endometrioid endometrial adenocarcinoma \\
\hline TCGA-EY-A1GW-01 & Primary tumor & Stage IVB & 337 & Endometrioid endometrial adenocarcinoma \\
\hline TCGA-EY-A1GU-01 & Primary tumor & Stage IB & 997 & Endometrioid endometrial adenocarcinoma \\
\hline TCGA-EY-A1GT-01 & Primary tumor & Stage IIIA & 490 & Endometrioid endometrial adenocarcinoma \\
\hline TCGA-EY-A1GR-01 & Primary tumor & Stage IA & 536 & Endometrioid endometrial adenocarcinoma \\
\hline TCGA-EY-A1GQ-01 & Primary tumor & Stage IB & 544 & Endometrioid endometrial adenocarcinoma \\
\hline TCGA-EY-A1GK-01 & Primary tumor & Stage IB & 693 & Endometrioid endometrial adenocarcinoma \\
\hline TCGA-EY-A1GJ-01 & Primary tumor & Stage IA & 727 & Endometrioid endometrial adenocarcinoma \\
\hline TCGA-EY-A1GI-01 & Primary tumor & Stage IB & 710 & Endometrioid endometrial adenocarcinoma \\
\hline TCGA-EY-A1GH-01 & Primary tumor & Stage IA & 710 & Endometrioid endometrial adenocarcinoma \\
\hline TCGA-EY-A1GF-01 & Primary tumor & Stage IA & 826 & Endometrioid endometrial adenocarcinoma \\
\hline TCGA-EY-A1GE-01 & Primary tumor & Stage IA & 741 & Endometrioid endometrial adenocarcinoma \\
\hline TCGA-EY-A1GD-01 & Primary tumor & Stage IA & 1639 & Endometrioid endometrial adenocarcinoma \\
\hline TCGA-EY-A1GC-01 & Primary tumor & Stage IB & 1647 & Endometrioid endometrial adenocarcinoma \\
\hline TCGA-EY-A1G8-01 & Primary tumor & Stage IB & 456 & Endometrioid endometrial adenocarcinoma \\
\hline TCGA-EY-A1G7-01 & Primary tumor & Stage ॥ & 189 & Endometrioid endometrial adenocarcinoma \\
\hline TCGA-AP-A1E4-01 & Primary tumor & Stage IB & 1555 & Endometrioid endometrial adenocarcinoma \\
\hline TCGA-AP-A1E3-01 & Primary tumor & Stage IIIC1 & 847 & Endometrioid endometrial adenocarcinoma \\
\hline TCGA-AP-A1E1-01 & Primary tumor & Stage IA & 1395 & Endometrioid endometrial adenocarcinoma \\
\hline TCGA-AP-A1E0-01 & Primary tumor & Stage IIIC2 & 1832 & Endometrioid endometrial adenocarcinoma \\
\hline TCGA-AP-A1DV-01 & Primary tumor & Stage ॥ & 204 & Endometrioid endometrial adenocarcinoma \\
\hline TCGA-AP-A1DR-01 & Primary tumor & Stage $I I C 1$ & 1495 & Endometrioid endometrial adenocarcinoma \\
\hline TCGA-AP-A1DP-01 & Primary tumor & Stage IA & 1081 & Endometrioid endometrial adenocarcinoma \\
\hline TCGA-AP-A1DO-01 & Primary tumor & Stage IA & 952 & Endometrioid endometrial adenocarcinoma \\
\hline TCGA-AP-A1DM-01 & Primary tumor & Stage IA & 1700 & Endometrioid endometrial adenocarcinoma \\
\hline TCGA-AP-A1DK-01 & Primary tumor & Stage IA & 2767 & Endometrioid endometrial adenocarcinoma \\
\hline TCGA-AP-A1DH-01 & Primary tumor & Stage IA & 2180 & Endometrioid endometrial adenocarcinoma \\
\hline TCGA-AX-A1CN-01 & Primary tumor & Stage IIB & 1143 & Endometrioid endometrial adenocarcinoma \\
\hline TCGA-AX-A1CK-11 & Primary tumor & Stage IB & 2441 & Endometrioid endometrial adenocarcinoma \\
\hline TCGA-AX-A1CJ-11 & Primary tumor & Stage IC & 2233 & Endometrioid endometrial adenocarcinoma \\
\hline TCGA-AX-A1Cl-01 & Primary tumor & Stage IB & 2600 & Endometrioid endometrial adenocarcinoma \\
\hline
\end{tabular}


Table 2 continued

\begin{tabular}{|c|c|c|c|c|}
\hline Sample ID & Sample_type & Clinical_stage & _os & Histological_type \\
\hline TCGA-AX-A1CF-01 & Primary tumor & Stage IVB & 2337 & Endometrioid endometrial adenocarcinoma \\
\hline TCGA-AX-A1CE-01 & Primary tumor & Stage IB & 1037 & Endometrioid endometrial adenocarcinoma \\
\hline TCGA-AX-A1C9-01 & Primary tumor & Stage IB & 802 & Endometrioid endometrial adenocarcinoma \\
\hline TCGA-AX-A1C5-01 & Primary tumor & Stage IIIC & 1001 & Endometrioid endometrial adenocarcinoma \\
\hline TCGA-AX-A1C4-01 & Primary tumor & Stage IB & 404 & Endometrioid endometrial adenocarcinoma \\
\hline TCGA-DI-A1BY-01 & Primary tumor & Stage ॥B & 1114 & Endometrioid endometrial adenocarcinoma \\
\hline TCGA-D1-A0ZV-01 & Primary tumor & Stage IB & 728 & Endometrioid endometrial adenocarcinoma \\
\hline TCGA-D1-A0ZU-01 & Primary tumor & Stage IB & 872 & Endometrioid endometrial adenocarcinoma \\
\hline TCGA-D1-A0ZS-01 & Primary tumor & Stage IB & 148 & Endometrioid endometrial adenocarcinoma \\
\hline TCGA-D1-A0ZR-01 & Primary tumor & Stage IB & 17 & Endometrioid endometrial adenocarcinoma \\
\hline TCGA-D1-A0ZQ-01 & Primary tumor & Stage IB & 260 & Endometrioid endometrial adenocarcinoma \\
\hline TCGA-D1-A0ZO-01 & Primary tumor & Stage IB & 602 & Endometrioid endometrial adenocarcinoma \\
\hline TCGA-D1-A0ZN-01 & Primary tumor & Stage IA & 512 & Endometrioid endometrial adenocarcinoma \\
\hline TCGA-BG-AOYU-01 & Primary tumor & Stage IA & 451 & Endometrioid endometrial adenocarcinoma \\
\hline TCGA-BS-A0WQ-01 & Primary tumor & Stage IA & 2022 & Endometrioid endometrial adenocarcinoma \\
\hline TCGA-DI-AOWH-01 & Primary tumor & Stage IB & 496 & Endometrioid endometrial adenocarcinoma \\
\hline TCGA-BG-A0W2-01 & Primary tumor & Stage IA & 1721 & Endometrioid endometrial adenocarcinoma \\
\hline TCGA-BG-A0W1-01 & Primary tumor & Stage ॥ & 1607 & Endometrioid endometrial adenocarcinoma \\
\hline TCGA-BG-A0VZ-01 & Primary tumor & Stage IIIA & 1601 & Endometrioid endometrial adenocarcinoma \\
\hline TCGA-BG-A0VX-01 & Primary tumor & Stage IB & 1043 & Endometrioid endometrial adenocarcinoma \\
\hline TCGA-BG-A0WW-01 & Primary tumor & Stage IA & 1582 & Endometrioid endometrial adenocarcinoma \\
\hline TCGA-BG-A0W-01 & Primary tumor & Stage IA & 1553 & Endometrioid endometrial adenocarcinoma \\
\hline TCGA-BG-A0VT-01 & Primary tumor & Stage IIIA & 1568 & Endometrioid endometrial adenocarcinoma \\
\hline TCGA-A5-A0VQ-01 & Primary tumor & Stage IA & 485 & Endometrioid endometrial adenocarcinoma \\
\hline TCGA-A5-A0VP-01 & Primary tumor & Stage IA & 1288 & Endometrioid endometrial adenocarcinoma \\
\hline TCGA-A5-A0VO-01 & Primary tumor & Stage IB & 875 & Endometrioid endometrial adenocarcinoma \\
\hline TCGA-BS-A0VI-01 & Primary tumor & Stage IIB & 2646 & Endometrioid endometrial adenocarcinoma \\
\hline TCGA-BS-A0V8-01 & Primary tumor & Stage ॥B & 2544 & Endometrioid endometrial adenocarcinoma \\
\hline TCGA-BS-A0V6-01 & Primary tumor & Stage IB & 2725 & Endometrioid endometrial adenocarcinoma \\
\hline TCGA-BS-AOUV-01 & Primary tumor & Stage IIIC & 2228 & Endometrioid endometrial adenocarcinoma \\
\hline TCGA-BS-AOUT-01 & Primary tumor & Stage IIB & 2212 & Endometrioid endometrial adenocarcinoma \\
\hline TCGA-BS-A0UM-01 & Primary tumor & Stage IB & 2644 & Endometrioid endometrial adenocarcinoma \\
\hline TCGA-BS-AOUL-01 & Primary tumor & Stage IA & 2481 & Endometrioid endometrial adenocarcinoma \\
\hline TCGA-BS-A0UJ-01 & Primary tumor & Stage IA & 2506 & Endometrioid endometrial adenocarcinoma \\
\hline TCGA-BS-AOUF-01 & Primary tumor & Stage IB & 2611 & Endometrioid endometrial adenocarcinoma \\
\hline TCGA-BS-AOUA-01 & Primary tumor & Stage IB & 3495 & Endometrioid endometrial adenocarcinoma \\
\hline TCGA-BS-A0U9-01 & Primary tumor & Stage IIA & 2825 & Endometrioid endometrial adenocarcinoma \\
\hline TCGA-BS-A0U8-01 & Primary tumor & Stage IIIC & 2963 & Endometrioid endometrial adenocarcinoma \\
\hline TCGA-BS-A0U7-01 & Primary tumor & Stage IC & 935 & Endometrioid endometrial adenocarcinoma \\
\hline TCGA-BS-A0U5-01 & Primary tumor & Stage IA & 2935 & Endometrioid endometrial adenocarcinoma \\
\hline
\end{tabular}


Table 2 continued

\begin{tabular}{|c|c|c|c|c|}
\hline Sample ID & Sample_type & Clinical_stage & -OS & Histological_type \\
\hline TCGA-BS-A0TJ-01 & Primary tumor & Stage IC & 2068 & Endometrioid endometrial adenocarcinoma \\
\hline TCGA-BS-A0TI-01 & Primary tumor & Stage IC & 1882 & Endometrioid endometrial adenocarcinoma \\
\hline TCGA-BS-A0TG-01 & Primary tumor & Stage IIIA & 2371 & Endometrioid endometrial adenocarcinoma \\
\hline TCGA-BS-A0TE-01 & Primary tumor & Stage IVB & 146 & Endometrioid endometrial adenocarcinoma \\
\hline TCGA-BS-A0TD-01 & Primary tumor & Stage IB & 2379 & Endometrioid endometrial adenocarcinoma \\
\hline TCGA-BS-A0TC-01 & Primary tumor & Stage IB & 2602 & Endometrioid endometrial adenocarcinoma \\
\hline TCGA-BS-A0TA-01 & Primary tumor & Stage IVB & 740 & Endometrioid endometrial adenocarcinoma \\
\hline TCGA-BS-A0T9-01 & Primary tumor & Stage IVB & 1428 & Endometrioid endometrial adenocarcinoma \\
\hline TCGA-BG-A0RY-01 & Primary tumor & Stage IB & 469 & Endometrioid endometrial adenocarcinoma \\
\hline TCGA-A5-A0RA-01 & Primary tumor & Stage IB & 884 & Endometrioid endometrial adenocarcinoma \\
\hline TCGA-A5-A0R9-01 & Primary tumor & Stage IA & 749 & Endometrioid endometrial adenocarcinoma \\
\hline TCGA-A5-A0R8-01 & Primary tumor & Stage IB & 596 & Endometrioid endometrial adenocarcinoma \\
\hline TCGA-A5-A0R7-01 & Primary tumor & Stage IA & 535 & Endometrioid endometrial adenocarcinoma \\
\hline TCGA-BG-A0MU-01 & Primary tumor & Stage IIIA & 617 & Endometrioid endometrial adenocarcinoma \\
\hline TCGA-BG-A0MT-01 & Primary tumor & Stage IA & 644 & Endometrioid endometrial adenocarcinoma \\
\hline TCGA-BG-A0MS-01 & Primary tumor & Stage $I I C 1$ & 1882 & Endometrioid endometrial adenocarcinoma \\
\hline TCGA-BG-A0MQ-01 & Primary tumor & Stage IA & 1817 & Endometrioid endometrial adenocarcinoma \\
\hline TCGA-BG-A0MO-01 & Primary tumor & Stage IA & 1309 & Endometrioid endometrial adenocarcinoma \\
\hline TCGA-BG-A0MI-01 & Primary tumor & Stage IA & 714 & Endometrioid endometrial adenocarcinoma \\
\hline TCGA-BG-A0MH-01 & Primary tumor & Stage IC & 1930 & Endometrioid endometrial adenocarcinoma \\
\hline TCGA-BG-A0MG-01 & Primary tumor & Stage IA & 1477 & Endometrioid endometrial adenocarcinoma \\
\hline TCGA-BG-A0MC-01 & Primary tumor & Stage IA & 1263 & Endometrioid endometrial adenocarcinoma \\
\hline TCGA-BG-A0MA-11 & Primary tumor & Stage IB & 326 & Endometrioid endometrial adenocarcinoma \\
\hline TCGA-BG-A0M9-01 & Primary tumor & Stage IB & 2270 & Endometrioid endometrial adenocarcinoma \\
\hline TCGA-BG-A0M8-01 & Primary tumor & Stage IA & 2020 & Endometrioid endometrial adenocarcinoma \\
\hline TCGA-BG-A0M7-01 & Primary tumor & Stage $I I C 1$ & 1937 & Endometrioid endometrial adenocarcinoma \\
\hline TCGA-BG-A0M4-01 & Primary tumor & Stage IA & 2167 & Endometrioid endometrial adenocarcinoma \\
\hline TCGA-BG-A0M3-01 & Primary tumor & Stage IB & 1071 & Endometrioid endometrial adenocarcinoma \\
\hline TCGA-BG-A0M2-01 & Primary tumor & Stage IB & 637 & Endometrioid endometrial adenocarcinoma \\
\hline TCGA-BG-A0M0-01 & Primary tumor & Stage IA & 588 & Endometrioid endometrial adenocarcinoma \\
\hline TCGA-BG-A0LX-01 & Primary tumor & Stage IB & 614 & Endometrioid endometrial adenocarcinoma \\
\hline TCGA-BG-A0LW-01 & Primary tumor & Stage IA & 566 & Endometrioid endometrial adenocarcinoma \\
\hline TCGA-AP-A0LV-01 & Primary tumor & Stage IA & 763 & Endometrioid endometrial adenocarcinoma \\
\hline TCGA-AP-A0LT-01 & Primary tumor & Stage II & 1497 & Endometrioid endometrial adenocarcinoma \\
\hline TCGA-AP-AOLS-01 & Primary tumor & Stage IA & 2904 & Endometrioid endometrial adenocarcinoma \\
\hline TCGA-AP-A0LQ-01 & Primary tumor & Stage IA & 2457 & Endometrioid endometrial adenocarcinoma \\
\hline TCGA-AP-A0LP-01 & Primary tumor & Stage IA & 1875 & Endometrioid endometrial adenocarcinoma \\
\hline TCGA-AP-A0LO-01 & Primary tumor & Stage IA & 1016 & Endometrioid endometrial adenocarcinoma \\
\hline TCGA-AP-A0LN-01 & Primary tumor & Stage IA & 2510 & Endometrioid endometrial adenocarcinoma \\
\hline TCGA-AP-A0LM-01 & Primary tumor & Stage $\| I C 2$ & 825 & Endometrioid endometrial adenocarcinoma \\
\hline
\end{tabular}


Table 2 continued

\begin{tabular}{|c|c|c|c|c|}
\hline Sample ID & Sample_type & Clinical_stage & _os & Histological_type \\
\hline TCGA-AP-AOLL-01 & Primary tumor & Stage IA & 2554 & Endometrioid endometrial adenocarcinoma \\
\hline TCGA-AP-A0LJ-01 & Primary tumor & Stage IA & 1421 & Endometrioid endometrial adenocarcinoma \\
\hline TCGA-AP-AOLG-01 & Primary tumor & Stage IA & 2015 & Endometrioid endometrial adenocarcinoma \\
\hline TCGA-AP-AOLF-01 & Primary tumor & Stage IVB & 2859 & Endometrioid endometrial adenocarcinoma \\
\hline TCGA-AP-A0LE-01 & Primary tumor & Stage IA & 3357 & Endometrioid endometrial adenocarcinoma \\
\hline TCGA-AP-A0LD-01 & Primary tumor & Stage IB & 3589 & Endometrioid endometrial adenocarcinoma \\
\hline TCGA-B5-A0KB-01 & Primary tumor & Stage IB & 824 & Endometrioid endometrial adenocarcinoma \\
\hline TCGA-B5-A0K7-01 & Primary tumor & Stage IA & 1933 & Endometrioid endometrial adenocarcinoma \\
\hline TCGA-B5-A0K6-01 & Primary tumor & Stage IA & 1953 & Endometrioid endometrial adenocarcinoma \\
\hline TCGA-B5-A0K4-01 & Primary tumor & Stage IB & 1757 & Endometrioid endometrial adenocarcinoma \\
\hline TCGA-B5-A0K3-01 & Primary tumor & Stage IA & 700 & Endometrioid endometrial adenocarcinoma \\
\hline TCGA-B5-A0K2-01 & Primary tumor & Stage IIIA & 2209 & Endometrioid endometrial adenocarcinoma \\
\hline TCGA-B5-A0K1-01 & Primary tumor & Stage IA & 1110 & Endometrioid endometrial adenocarcinoma \\
\hline TCGA-B5-A0K0-01 & Primary tumor & Stage IA & 1478 & Endometrioid endometrial adenocarcinoma \\
\hline TCGA-FL-A1YN-11 & Solid tissue normal & & & \\
\hline TCGA-FL-A1YU-11 & Solid tissue normal & & & \\
\hline TCGA-FL-A1YL-11 & Solid tissue normal & & & \\
\hline TCGA-FL-A1YI-11 & Solid tissue normal & & & \\
\hline TCGA-AX-A0J0-11 & Solid tissue normal & & & \\
\hline TCGA-FL-A1YQ-11 & Solid tissue normal & & & \\
\hline TCGA-FL-A1YF-11 & Solid tissue normal & & & \\
\hline TCGA-FL-A3WE-11 & Solid tissue normal & & & \\
\hline TCGA-FL-A1YV-11 & Solid tissue normal & & & \\
\hline TCGA-FL-A1YT-11 & Solid tissue normal & & & \\
\hline TCGA-AJ-A3NC-11 & Solid tissue normal & & & \\
\hline TCGA-AJ-A3NE-11 & Solid tissue normal & & & \\
\hline TCGA-AX-A2HC-11 & Solid tissue normal & & & \\
\hline TCGA-AX-A2HD-11 & Solid tissue normal & & & \\
\hline TCGA-BK-A4ZD-11 & Solid tissue normal & & & \\
\hline TCGA-BG-A3EW-11 & Solid tissue normal & & & \\
\hline TCGA-FL-A1YH-11 & Solid tissue normal & & & \\
\hline TCGA-AJ-A3NH-11 & Solid tissue normal & & & \\
\hline TCGA-AX-A05Y-11 & Solid tissue normal & & & \\
\hline TCGA-DI-A2QY-11 & Solid tissue normal & & & \\
\hline TCGA-BG-A2AD-11 & Solid tissue normal & & & \\
\hline TCGA-FL-A1YG-11 & Solid tissue normal & & & \\
\hline TCGA-AX-A0IZ-11 & Solid tissue normal & & & \\
\hline TCGA-BG-A3PP-11 & Solid tissue normal & & & \\
\hline TCGA-AX-A2H5-01 & Solid tissue normal & & & \\
\hline TCGA-AX-A2H4-11 & Solid tissue normal & & & \\
\hline
\end{tabular}


Table $\mathbf{2}$ continued

\begin{tabular}{|c|c|c|c|c|}
\hline Sample ID & Sample_type & Clinical_stage & _OS & Histological_type \\
\hline TCGA-DI-A1NN-01 & Solid tissue normal & & & \\
\hline TCGA-EO-A22T-01 & Solid tissue normal & & & \\
\hline TCGA-EO-A22S-01 & Solid tissue normal & & & \\
\hline TCGA-EO-A22R-01 & Solid tissue normal & & & \\
\hline TCGA-BK-A13C-01 & Solid tissue normal & & & \\
\hline TCGA-DI-A2QU-01 & Solid tissue normal & & & \\
\hline TCGA-AJ-A2QL-01 & Solid tissue normal & & & \\
\hline TCGA-BG-A2L7-01 & Solid tissue normal & & & \\
\hline TCGA-AX-A2HA-01 & Solid tissue normal & & & \\
\hline TCGA-AX-A2H8-01 & Solid tissue normal & & & \\
\hline TCGA-AX-A2H7-01 & Solid tissue normal & & & \\
\hline TCGA-BG-A2AE-01 & Solid tissue normal & & & \\
\hline TCGA-DI-A1NO-01 & Solid tissue normal & & & \\
\hline TCGA-E6-A1M0-01 & Solid tissue normal & & & \\
\hline TCGA-AX-A1CK-01 & Solid tissue normal & & & \\
\hline TCGA-AX-A1CJ-01 & Solid tissue normal & & & \\
\hline TCGA-AX-A1Cl-11 & Solid tissue normal & & & \\
\hline TCGA-AX-A1CF-11 & Solid tissue normal & & & \\
\hline TCGA-BG-A0MA-01 & Solid tissue normal & & & \\
\hline TCGA-BK-AOCB-01 & Solid tissue normal & & & \\
\hline TCGA-FL-A1YM-11 & Solid tissue normal & & & \\
\hline TCGA-FL-A1YN-11 & Solid tissue normal & & & \\
\hline TCGA-FL-A1YU-11 & Solid tissue normal & & & \\
\hline TCGA-FL-A1YL-11 & Solid tissue normal & & & \\
\hline TCGA-FL-A1YI-11 & Solid tissue normal & & & \\
\hline TCGA-AX-A0J0-11 & Solid tissue normal & & & \\
\hline TCGA-FL-A1YQ-11 & Solid tissue normal & & & \\
\hline TCGA-FL-A1YF-11 & Solid tissue normal & & & \\
\hline TCGA-FL-A3WE-11 & Solid tissue normal & & & \\
\hline TCGA-FL-A1YV-11 & Solid tissue normal & & & \\
\hline TCGA-FL-A1YT-11 & Solid tissue normal & & & \\
\hline TCGA-AJ-A3NC-11 & Solid tissue normal & & & \\
\hline TCGA-AJ-A3NE-11 & Solid tissue normal & & & \\
\hline TCGA-AX-A2HC-11 & Solid tissue normal & & & \\
\hline TCGA-AX-A2HD-11 & Solid tissue normal & & & \\
\hline TCGA-BK-A4ZD-11 & Solid tissue normal & & & \\
\hline TCGA-BG-A3EW-11 & Solid tissue normal & & & \\
\hline TCGA-FL-A1YH-11 & Solid tissue normal & & & \\
\hline TCGA-AJ-A3NH-11 & Solid tissue normal & & & \\
\hline TCGA-AX-A05Y-11 & Solid tissue normal & & & \\
\hline
\end{tabular}


Table 2 continued

\begin{tabular}{|c|c|c|c|c|}
\hline Sample ID & Sample_type & Clinical_stage & _os & Histological_type \\
\hline TCGA-DI-A2QY-11 & Solid tissue normal & & & \\
\hline TCGA-BG-A2AD-11 & Solid tissue normal & & & \\
\hline TCGA-FL-A1YG-11 & Solid tissue normal & & & \\
\hline TCGA-AX-A0IZ-11 & Solid tissue normal & & & \\
\hline TCGA-BG-A3PP-11 & Solid tissue normal & & & \\
\hline TCGA-AX-A2H5-01 & Solid tissue normal & & & \\
\hline TCGA-AX-A2H4-11 & Solid tissue normal & & & \\
\hline TCGA-DI-A1NN-01 & Solid tissue normal & & & \\
\hline TCGA-EO-A22T-01 & Solid tissue normal & & & \\
\hline TCGA-EO-A22S-01 & Solid tissue normal & & & \\
\hline TCGA-EO-A22R-01 & Solid tissue normal & & & \\
\hline TCGA-BK-A13C-01 & Solid tissue normal & & & \\
\hline TCGA-DI-A2QU-01 & Solid tissue normal & & & \\
\hline TCGA-AJ-A2QL-01 & Solid tissue normal & & & \\
\hline TCGA-BG-A2L7-01 & Solid tissue normal & & & \\
\hline TCGA-AX-A2HA-01 & Solid tissue normal & & & \\
\hline TCGA-AX-A2H8-01 & Solid tissue normal & & & \\
\hline TCGA-AX-A2H7-01 & Solid tissue normal & & & \\
\hline TCGA-BG-A2AE-01 & Solid tissue normal & & & \\
\hline TCGA-DI-A1NO-01 & Solid tissue normal & & & \\
\hline TCGA-E6-A1M0-01 & Solid tissue normal & & & \\
\hline TCGA-AX-A1CK-01 & Solid tissue normal & & & \\
\hline TCGA-AX-A1CJ-01 & Solid tissue normal & & & \\
\hline TCGA-AX-A1CI-11 & Solid tissue normal & & & \\
\hline TCGA-AX-A1CF-11 & Solid tissue normal & & & \\
\hline
\end{tabular}

investigate whether E2F3 is a direct target gene of HOXB9, we performed chromatin immunoprecipitation (ChIP) assays. Interestingly, the results showed that HOXB9 binds directly to the promoter of E2F3 (Fig. 4d). Moreover, we extracted the protein in the mice tumor in Fig. 2d, and analyzed the protein levels by western blot with the indicated antibodies. Consistently, both HOXB9 and E2F3 protein levels were decreased in the tumor with lentiviruses carrying HOXB9 shRNA, compared with control shRNA (Fig. S1g). Therefore, these results indicated that E2F3 is a direct downstream target of HOXB9.

\section{Knocking down E2F3 abolished the ability of HOXB9 in enhancing cancer cell migration}

To further elucidate the role of HOXB9 in regulating EC cell migration mediated by E2F3, we inhibited endogenous E2F3 in EC cell line Ishikawa with E2F3 siRNA
(Fig. 5a). The transwell assays showed that knocked down E2F3 caused a significant decrease of Ishikawa cell migration (Figs. 5b, c). Furthermore, we found that knocking down E2F3 abolished the ability of HOXB9 in enhancing Ishikawa cell migration (Figs. $5 \mathrm{~d}$, e). Moreover, we established the Ishikawa stable cell lines infected with lentiviruses carrying E2F3 shRNA. However, using transwell assays, we found overexpression of HOXB9 did not increased cell migration ability in sh-E2F3 cells (Fig. 5f). Next, we overexpressed E2F3 shRNA resistant mutant plasmid in sh-E2F3 cells with the recovery of the E2F3 expression. Interestingly, we found that overexpression of E2F3 shRNA-resistant mutant plasmid increased cell migration ability. Surprisingly, after overexpression of HOXB9 in the cells with recovery of E2F3 expression, the cell migration ability markedly increased, compared with the vector group (Fig. 5f). In addition, the 


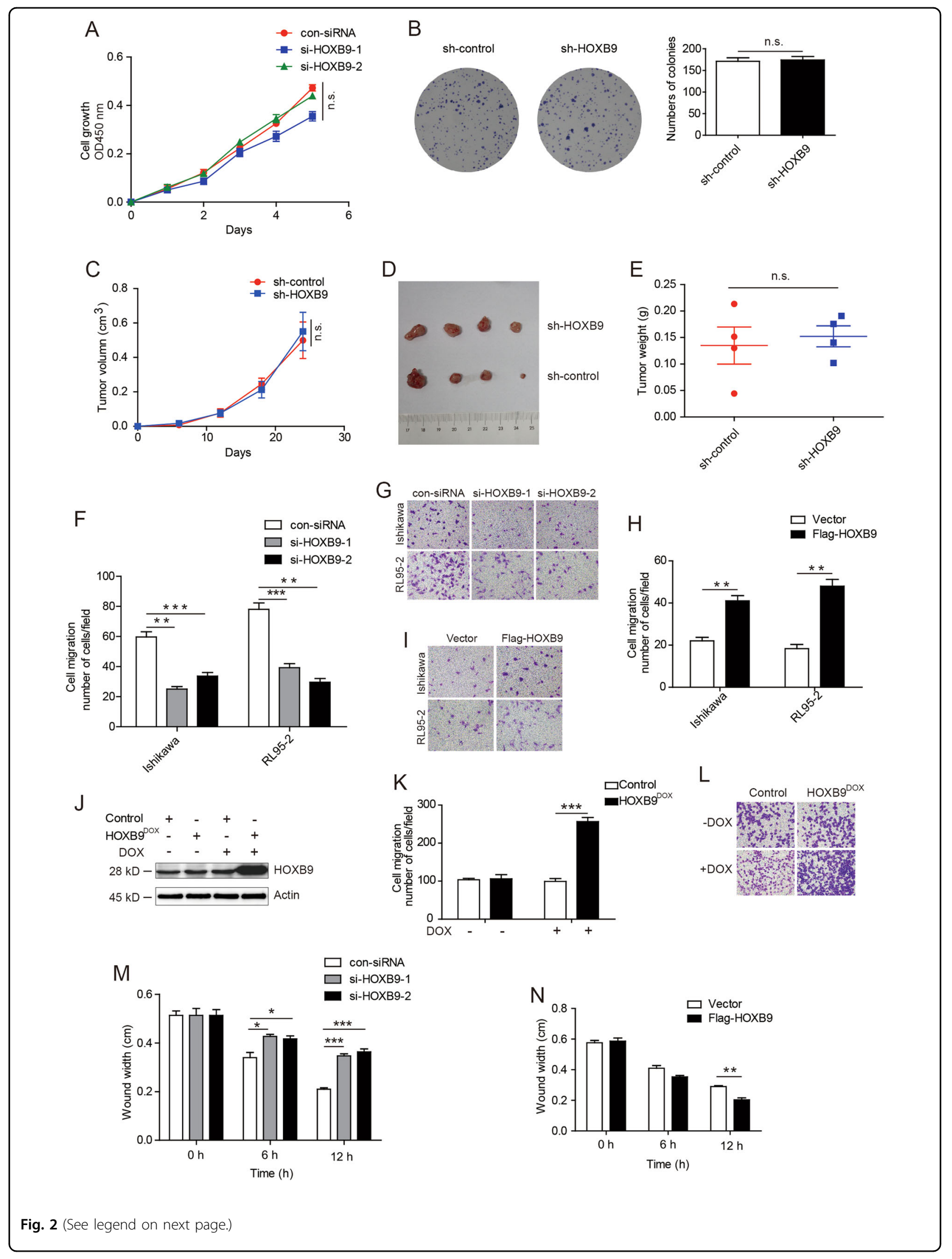




\begin{abstract}
(see figure on previous page)
Fig. 2 HOXB9 promotes endometrial cancer cell migration. a Cell proliferation assays were performed in endometrial cancer cell line Ishikawa, which was knocked down with two different HOXB9 small interfering RNA (siRNA). $\mathbf{b}$ The colony formation assays were performed using the Ishikawa stable cell lines infected with lentiviruses carrying control shRNA or HOXB9 shRNA. Cells were incubated for 2 weeks, and washed three times with PBS and fixed with $4 \%$ paraformaldehyde, following with staining by $0.5 \%$ crystal violet solution. c The mouse xenograft assays using the Ishikawa stable cell lines infected with lentiviruses carrying control shRNA or HOXB9 shRNA. Tumor growths in xenografted nude mice were measured and plotted. $\mathbf{d}$ The xenograft tumors were dissected and photographed at day 24. e Average tumor weights were measured at day 24. $\mathbf{f}$-h The effect of HOXB9 on cell migration in Ishikawa and RL95-2 was determined. Data were presented as mean \pm SEM from three independent experiments. Representative images of cell migration in Ishikawa and RL95-2 were shown. $\mathbf{j}$ Western blot was performed to detect the expression of HOXB9 by adding doxycycline. $\mathbf{k}$ The transwell assays were performed by adding doxycycline to induce HOXB9 expression. Data were presented as mean \pm SEM from three independent experiments. I Representative images of cell migration were shown. $\mathbf{m}, \mathbf{n}$ The wound-healing assays were performed in Ishikawa cells with knocked down or overexpressed of HOXB9, and images were taken at 0,6 and $12 \mathrm{~h}$ after wound. The wound widths were measured and quantified. ${ }^{*} P<0.05,{ }^{*} P<0.01,{ }^{* * *} P<0.001$
\end{abstract}

wound-healing assay results indicated that, after inhibiting E2F3, overexpressing HOXB9 did not affect the Ishikawa cell migration (Fig. 5g). Collectively, these findings showed E2F3 is an important downstream effector of HOXB9 in regulating EC cell migration.

\section{Discussion}

$\mathrm{EC}$ is one of the most common gynecologic malignancy in the whole world ${ }^{1,2,5}$. From 1987 to 2008, the incidence of EC increased $50 \%$, and the number of associated deaths increased approximately $300 \%$. In 2017, an estimated 61,380 women in the United States were diagnosed with EC, and almost 11,000 died from this disease. Although there have been some chemotherapeutic and targeted therapy agents treated for ovarian, fallopian tube and primary peritoneal cancers, little therapy agents were effective for the palliative treatment of advanced $\mathrm{EC}^{36,37}$. Therefore, this highlights the need for understanding the molecular and biochemical mechanisms in advanced, recurrent, metastatic EC, and to develop new therapeutic approaches. Although there have been reports clarifying some signaling pathways in $\mathrm{EC}^{38-43}$, there were no reports about the relationship between HOXB9 and EC.

HOXB9 belongs to HOX family member. It mainly functions as a transcription factor, which plays important roles in embryo development and cancer progression ${ }^{17,19-}$ 21,44 . The role of HOXB9 in cancer progression is complicated $^{15,16,45,46}$. HOXB9 was found to be overexpressed in lung adenocarcinoma and human breast cancer, whereas in colon adenocarcinoma, pancreatic ductal adenocarcinoma and gastric carcinoma patients, its expression is decreased ${ }^{15-19,21}$. In this study, we found the expression of HOXB9 in endometrial carcinoma is higher than normal proliferative endometrium and atypical endometrial hyperplasia. In addition, HOXB9 expression notably correlated with histological grade and lymph node metastasis status. The results using immunohistochemical staining and bioinformatics analysis in TCGA EC database showed that elevated HOXB9 expression level corresponds to a poor overall survival in EC. Knocking down with HOXB9 siRNA only inhibits EC cell migration, but not cell proliferation, which partially explained why HOXB9 plays oncogenic role in EC progression.

HOXB9 was previously reported to regulate cancer progression, by targeting EMT via the transforming growth factor- $\beta 1 /$ Smad2/Slug signaling pathway ${ }^{47}$. We also found HOXB9 regulated lung adenocarcinoma progression by targeting oncogenic protein JMJD6 ${ }^{18}$. However, the mechanism underlying HOXB9 in EC progression remains unknown. Using bioinformatics analysis in TCGA EC database, we found many genes co-expressed with HOXB9. In addition, GO and KEGG analysis indicated these genes participated in multiple cancer-related signaling pathways. We picked the tumor-related protein E2F3. We found a positive correlation between HOXB9 and E2F3 mRNA expression levels. What's more, knocked down HOXB9 inhibited the expression of E2F3 both in protein and mRNA levels. The ChIP assay showed E2F3 is a direct downstream target of HOXB9. In transwell and wound-healing assays, knocking down E2F3 abolished the ability of HOXB9 in enhancing cancer cell migration. These findings showed E2F3 is an important downstream effector of HOXB9 in regulating EC cell migration.

In summary, we have demonstrated that HOXB9 expression is increased in EC patients, and HOXB9 promotes EC cell migration by regulating E2F3 expression. Thus, HOXB9 may represent a prognostic marker and a potential therapeutic target for EC. However, the generic role of HOXB9 in vivo should be investigated in the future using transgenic mice models of HOXB9.

\section{Materials and methods Cell culture}

Human EC cell lines, Ishikawa and RL95-2, were purchased from ATCC (American Type Culture Collection). All cells were grown in Dulbecco's modified Eagle's medium supplemented with $10 \%$ fetal bovine serum (FBS) and cultured at $37^{\circ} \mathrm{C}$ with $5 \% \mathrm{CO}_{2}$. 


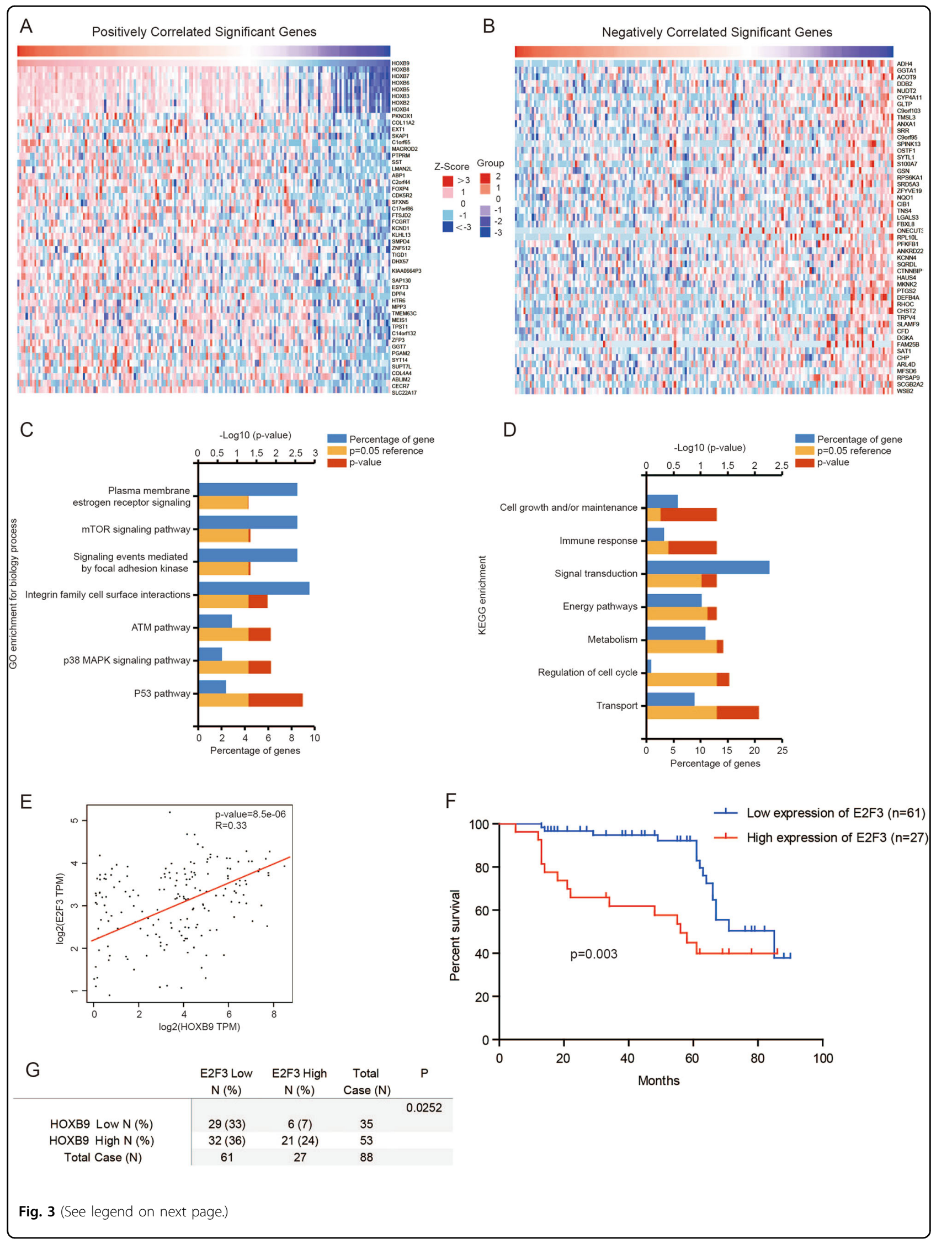


(see figure on previous page)

Fig. 3 The expression between HOXB9 and E2F3 is positively correlated. $\mathbf{a}$, $\mathbf{b}$ Genes highly co-expressed with HOXB9 in TCGA from the LinkedOmics database (http://www.linkedomics.org) were selected, and the top 50 positively a and 50 negatively $\mathbf{b}$ correlated genes are shown. c, d Gene ontology (GO) and Kyoto Encyclopedia of Genes and Genomes (KEGG) enrichment analysis on the correlated expressed genes. e Pearson correlation analysis revealed that E2F3 were significantly correlated with HOXB9 in human endometrial cancer using TCGA database. $\mathbf{f}$ The Kaplan-Meier analysis of E2F3 expression in 88 endometrial carcinoma was performed. $\mathbf{g}$ The correlation of HOXB9 and E2F3 expression in 88 endometrial carcinoma were analyzed

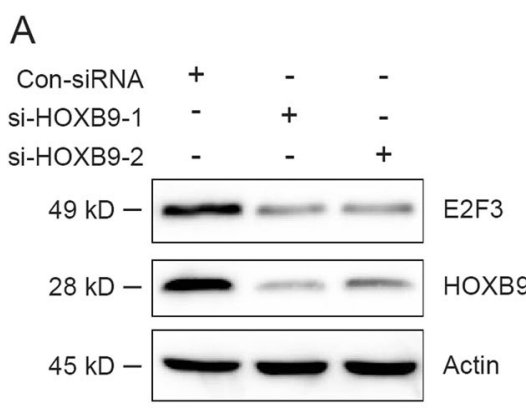

C

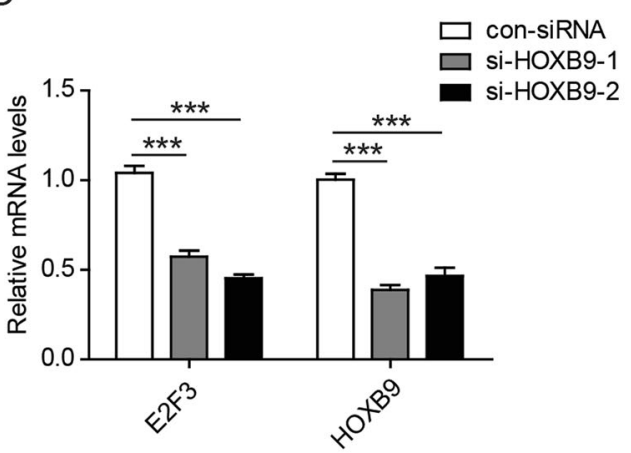

B

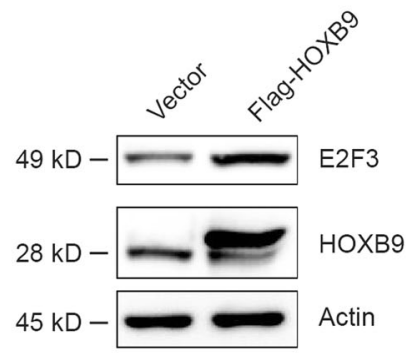

D

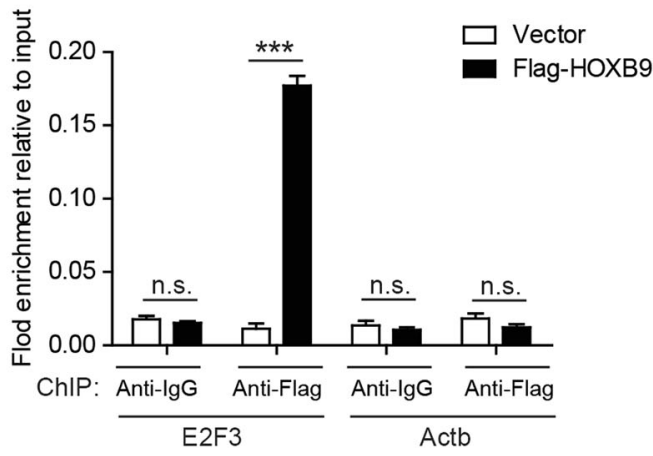

Fig. 4 E2F3 is a direct downstream target of HOXB9. a The endometrial cancer cell line Ishikawa was knocked down with two different HOXB9 small interfering RNA (siRNA), and cell lysates were analyzed by western blot with the indicated antibodies. $\mathbf{b}$ The endometrial cancer cell line Ishikawa was transfected with Flag-HOXB9 or empty vector plasmid, and cell lysates were analyzed by western blot with the indicated antibodies. c The endometrial cancer cell line Ishikawa was knocked down with two different HOXB9 siRNA, and E2F3 and HOXB9 mRNA expression levels were analyzed by real-time PCR. $\mathbf{d}$ ChIP assays were performed using control IgG or anti-Flag antibody in Ishikawa cells. The binding of HOXB9 on promoters of E2F3 and the negative control Actb genes were analyzed by real-time PCR. Data were presented as mean \pm SEM from three independent experiments. The statistical analyses were performed by Student's $t$-test, ${ }^{* * *} P<0.001$

\section{Construction and antibodies}

Flag-HOXB9 expression plasmid was constructed by subcloning the HOXB9 full-length complementary DNA (cDNA) fragments into $3 \times$ flag vector. All constructs were confirmed by DNA sequencing. The following antibodies were used: HOXB9 (Santa Cruz: sc-398500 and sc-133671) and E2F3 (Abcam: ab50917).

\section{Cell proliferation assays}

Cell proliferation assays were performed using the CellTiter $96^{\circledR}$ AQueous One Solution Cell Proliferation Assay kit according to manufacturer's instructions
(Promega). Briefly, EC cells were plated in 96-well plates at a density of 900 cells per well. In all, $15 \mu \mathrm{l}$ CellTiter $96^{\circledR}$ AQueous One Solution reagent was added to the cells per well, and incubated for $1 \mathrm{~h}$ at $37^{\circ}$ C. Then cell growth was measured in a microplate reader at $490 \mathrm{~nm}$.

\section{Colony formation assay}

Colony formation assay was used to determine the function of HOXB9 in promoting cell proliferation. In brief, 2000 cells were seeded onto six-well culture plates. After incubation for 2 weeks, cells were washed three 

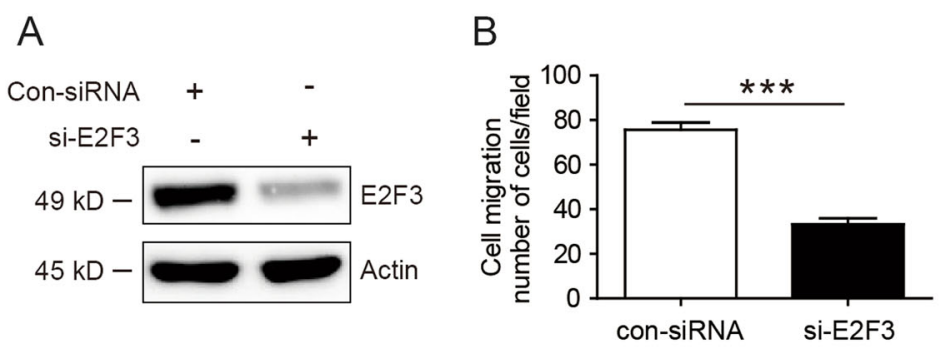

C

$\mathrm{D}$

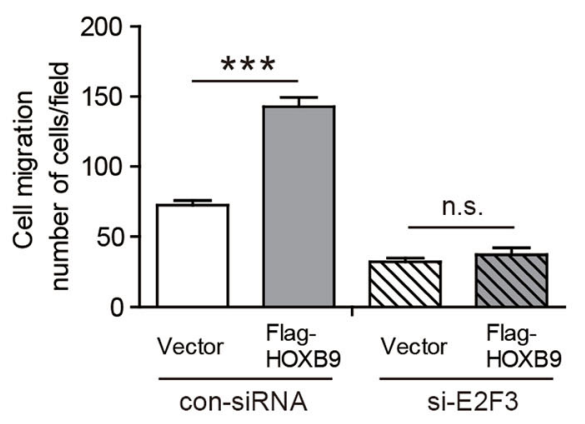

$\mathrm{F}$

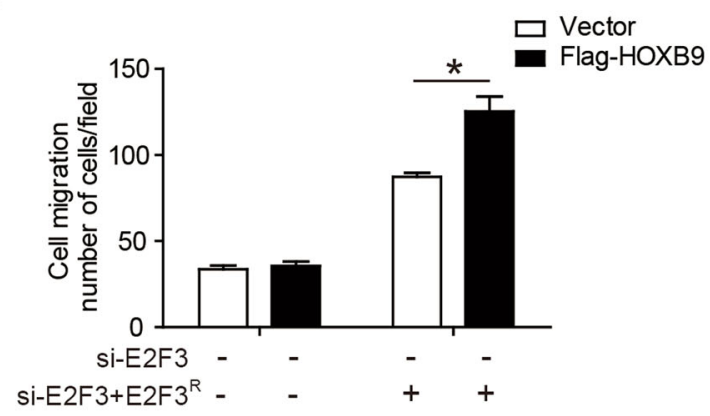

$\mathrm{E}$

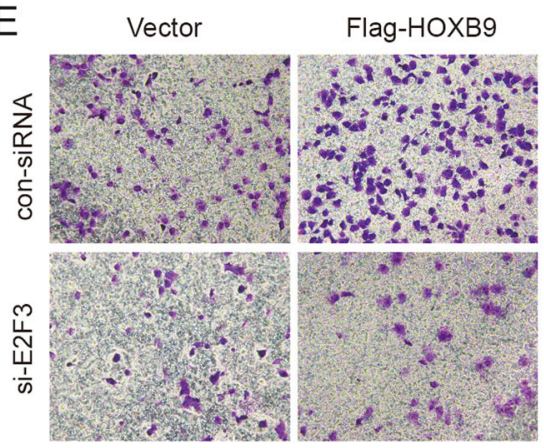

G

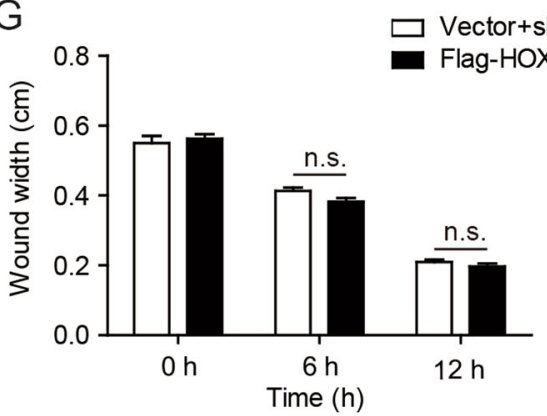

Fig. 5 Knocking down E2F3 abolished the ability of HOXB9 in enhancing cancer cell migration. a-c Ishikawa was knocked down with E2F3 small interfering RNA (siRNA) $\mathbf{a}$, and the effect of E2F3 on cell migration in Ishikawa was determined $\mathbf{b}$. Data were presented as mean \pm SEM from three independent experiments. The representative images of cell migration in Ishikawa were shown at the right panel $\mathbf{c}$. $\mathbf{d}$, e Ishikawa was firstly transfected with Flag-HOXB9, then knocked down with E2F3 siRNA, and the cell migration in Ishikawa was determined $\mathbf{d}$. The representative images of cell migration in Ishikawa were shown at the right panel e. $\mathbf{f}$ We established the Ishikawa stable cell lines infected with lentiviruses carrying E2F3 shRNA. Next, we overexpressed E2F3 shRNA-resistant mutant plasmid in sh-E2F3 cells with the recovery of the E2F3 expression. Then, we overexpressed HOXB9 to detect the cell migration using transwell assays. $\mathbf{g}$ Ishikawa was first transfected with Flag-HOXB9, then knocked down with E2F3 siRNA, followed with wound-healing assays, and images were taken at 0,6 and $12 \mathrm{~h}$ after wound. The wound widths were measured and quantified. The statistical analyses were performed by Student's t-test, ${ }^{*}<0.05,{ }^{* *} \mathrm{P}<0.001$

times with phosphate-buffered saline (PBS) and fixed with $4 \%$ paraformaldehyde, following with staining by $0.5 \%$ crystal violet solution. The colonies were counted using a light microscope.

\section{In vivo xenograft tumor growth experiments}

Balb/c nude mice was injected subcutaneously into the flank with $1 \times 10^{6}$ Ishikawa stable cells. Tumor sizes were measured at the indicated time. After 24 days when the tumor reached to approximately $1 \mathrm{~cm}$ in diameter, the tumors were dissected, and the tumor weight was measured.

\section{Cell migration assays}

Cell suspension containing $1 \times 10^{5}$ cells $/ \mathrm{ml}$ was seed into the upper chamber in serum-free media. The lower wells contains $20 \%$ (v/v) FBS. After 8 -h migration at $37^{\circ} \mathrm{C}$, cells on the upper surface of the membrane filter were removed. The migrated cells through the inserts were fixed with $4 \%$ formaldehyde and stained by crystal violet, and counted.

\section{Wound-healing assays}

The EC cell lines were cultured until a monolayer forms. Artificial wounds were created on the cell 
monolayer, then capture the images of wound healing at 0,6 and $12 \mathrm{~h}$, and compare the images to quantify the cell migration rate.

\section{Real-time PCR}

Total RNA was isolated from cells using Trizol reagent (Invitrogen) and cDNA was synthesized. Two-step realtime polymerase chain reaction (PCR) was performed using the SYBR Green Mix (Roche) and a LightCycler ${ }^{\circledR} 96$ detection system (Roche) according to manufacturer's instructions. Specific primers for HOXB9 and E2F3 were designed as follows:

HOXB9 forward primer, 5'-CCATTTCTGGGACGCTTAGCA-3'; HOXB9 reverse primer, 5'-TGTAAGGGT GGTAGACGGACG-3'; E2F3 forward primer, 5'-AAGA AGAAGTCTAAAAACAACGTCCAA-3'; E2F3 reverse primer, 5'-CTTGACACTGGGCCAGCAT-3'.

All mRNAs were normalized to the mRNA level of GAPDH gene.

\section{ChIP assays}

The EC cell lines were crosslinked with $1 \%$ formaldehyde for $10 \mathrm{~min}$, and subjected to ChIP assays as described before ${ }^{48}$. The ChIP DNA complex were precipitated and subjected to RT-PCR with the indicated primers.

\section{Bioinformatics analysis of TCGA database}

The results of TCGA database were mainly analyzed by the web-based tools, GEPIA (http://gepia.cancer-pku.cn/) $)^{31}$ and the LinkedOmics database (http://www.linkedomics. org $)^{32}$, which are web-based tools to deliver fast and customizable functionalities based on TCGA data. GEPIA can deliver fast and customizable functionalities based on TCGA data, and provides key interactive and customizable functions including differential expression analysis, correlation analysis and patient survival analysis. The LinkedOmics web allows flexible exploration of associations between a molecular or clinical attribute of interest and all other attributes, providing the opportunity to analyze and visualize associations between billions of attribute pairs for each cancer cohort.

\section{Immunohistochemical staining}

The endometrial carcinoma were obtained from surgical excision in the third affiliated hospital of Zhengzhou University. According to age, the endometrial carcinoma were divided into: $\leq 50,41$ cases; $>50,47$ cases. Surgical staging: stage I, 53 cases; stage II, 18 cases; stage III-IV, 17 cases. Histological grade: G1, 29 cases; G2, 26 cases; G3, 33 cases. Muscular invasion endomembrane: $\leq 1 / 2,39$ cases; $>1 / 2,49$ cases. Lymph node metastisis: Yes, 39 cases; No, 49 cases. Normal endometrial samples were obtained from premenopausal women awaiting in vitro fertilization treatment. All of the above cases were treated for the first time. No chemotherapy, radiotherapy and other adjuvant treatment had been done before the operation. The atypical endometrial hyperplasia was characterized by numerous glands with irregular contour, papillary intraglandular proliferations, stratified epithelium (2-4 lines), with the loss of polarity and marked nuclear atypia, also presenting atypical mitoses. The glands were extremely irregular with respect to shape and size, arranged "back-to-back". However, the endometrial carcinoma were characterized by a glandular proliferation marked with intra-luminal papillary projections, with the presence of outgrowths and branches that realize a confluent pattern. These are lined by stratified neoplastic epithelia with a thin fibrovascular axis.

Immunohistochemical staining was performed using 5-mmol/L thick sections. Tissue sections were deparaffinized and rehydrated gradually, and antigen retrieval was performed in $\mathrm{pH} 6.0$ citrate buffer for $10 \mathrm{~min}$. To quench endogenous peroxidases, peroxide blocking was performed for $30 \mathrm{~min}$ with $3 \% \mathrm{H}_{2} \mathrm{O}_{2}$. The slides were incubated with primary antibody at $4{ }^{\circ} \mathrm{C}$ overnight, then the two-step plus poly-HRP anti-rabbit/mouse IgG detection system was applied. The immunohistochemical staining results were assigned a mean score considering both the intensity of staining and the proportion of cells with an unequivocal positive reaction. Each section was independently assessed by two pathologists without prior knowledge of patient data. Positive reactions were defined as those showing brown signals mainly in the cell nucleus. A staining index (values, $0-3$ ) was determined by the staining intensity and positive area. The scores $(0-3)$ were defined as follows: 0 , negative; 1 , weak; 2 , moderate; and 3 , strong. For statistical analysis, scores of $0-1$ were considered low expression and scores of 2-3 considered high expression.

\section{Statistical analysis}

Chi-square tests were used to determine category variable differences. Overall survival distributions were estimated using Kaplan-Meier analysis. For other experiments, statistical significance was determined by Student's $t$-test.

\section{Acknowledgements}

This study was supported by grants from the National Natural Science Foundation of China grants (grant no. 81702780) and the Youth Innovation Fund of the First Affiliated Hospital of Zhengzhou University.

\section{Author details}

${ }^{1}$ Department of Clinical Laboratory, The First Affiliated Hospital of Zhengzhou University, 450052 Zhengzhou, Henan, China. ${ }^{2}$ Department of Obstetrics and Gynecology, The Third Affiliated Hospital of Zhengzhou University, 450052 Zhengzhou, Henan, China. ${ }^{3}$ Department of General Surgery, Zhecheng People's Hospital, 476000 Shangqiu, Henan, China 


\section{Conflict of interest}

The authors declare that they have no conflict of interest.

\section{Publisher's note}

Springer Nature remains neutral with regard to jurisdictional claims in published maps and institutional affiliations.

Supplementary Information accompanies this paper at (https://doi.org/ 10.1038/s41419-018-0556-3).

Received: 25 December 2017 Revised: 30 March 2018 Accepted: 3 April 2018

Published online: 03 May 2018

\section{References}

1. Di Cristofano, A. \& Ellenson, L. H. Endometrial carcinoma. Annu Rev. Pathol. 2, 57-85 (2007).

2. Siegel, R. L., Miller, K. D. \& Jemal, A. Cancer statistics, 2017. CA Cancer J. Clin. 67, 7-30 (2017).

3. Martinez-Garcia, E. et al. Advances in endometrial cancer protein biomarkers for use in the clinic. Expert Rev. Proteom. 15, 81-99 (2017).

4. Lacey, J. V. Jr. \& Chia, V. M. Endometrial hyperplasia and the risk of progression to carcinoma. Maturitas 63, 39-44 (2009).

5. Makker, V. et al. New therapies for advanced, recurrent, and metastatic endometrial cancers. Gynecol. Oncol. Res. Pract. 4, 19 (2017).

6. Gehring, W. J., Affolter, M. \& Burglin, T. Homeodomain proteins. Annu. Rev. Biochem. 63, 487-526 (1994).

7. Apiou, F. et al. Fine mapping of human HOX gene clusters. Cytogenet. Cell Genet. 73, 114-115 (1996).

8. Garcia-Fernandez, J. The genesis and evolution of homeobox gene clusters. Nat. Rev. Genet. 6, 881-892 (2005).

9. Yahagi, N. et al. Position-specific expression of Hox genes along the gastrointestinal tract. Congenit. Anom. (Kyoto) 44, 18-26 (2004).

10. Kmita, M. et al. Early developmental arrest of mammalian limbs lacking HoxA HoxD gene function. Nature 435, 1113-1116 (2005).

11. Lewis, E. B. A gene complex controlling segmentation in Drosophila. Nature 276, 565-570 (1978)

12. Chen, F. \& Capecchi, M. R. Paralogous mouse Hox genes, Hoxa9, Hoxb9, and Hoxd9, function together to control development of the mammary gland in response to pregnancy. Proc. Natl. Acad. Sci. USA 96, 541-546 (1999).

13. Sakiyama, J., Yokouchi, Y. \& Kuroiwa, A. Coordinated expression of Hoxb genes and signaling molecules during development of the chick respiratory tract. Dev. Biol. 227, 12-27 (2000).

14. Zhan, J. et al. High expression of transcriptional factor HoxB9 predicts poor prognosis in patients with lung adenocarcinoma. Histopathology 66, 955-965 (2015).

15. Zhan, J. et al. Elevated HOXB9 expression promotes differentiation and predicts a favourable outcome in colon adenocarcinoma patients. Br. J. Cancer 111, 883-893 (2014)

16. Chang, Q. et al. HOXB9 induction of mesenchymal-to-epithelial transition in gastric carcinoma is negatively regulated by its hexapeptide motif. Oncotarget 6, 42838-42853 (2015).

17. Sha, S. et al. Decreased expression of HOXB9 is related to poor overall survival in patients with gastric carcinoma. Dig. Liver Dis. 45, 422-429 (2013).

18. Wan, J. et al. PCAF-mediated acetylation of transcriptional factor HOXB9 suppresses lung adenocarcinoma progression by targeting oncogenic protein JMJD6. Nucleic Acids Res. 44, 10662-10675 (2016).

19. Hayashida, T. et al. HOXB9, a gene overexpressed in breast cancer, promotes tumorigenicity and lung metastasis. Proc. Natl. Acad. Sci. USA 107, 1100-1105 (2010).

20. Chiba, N. et al. Homeobox B9 induces epithelial-to-mesenchymal transitionassociated radioresistance by accelerating DNA damage responses. Proc. Natl. Acad. Sci. USA 109, 2760-2765 (2012).

21. Shrestha, B. et al. Homeodomain-containing protein HOXB9 regulates expression of growth and angiogenic factors, facilitates tumor growth in vitro and is overexpressed in breast cancer tissue. FEBS J. 279, 3715-3726 (2012).
22. Ceol, C. J. \& Horvitz, H. R. dpl-1 DP and efl-1 E2F act with lin-35 Rb to antagonize Ras signaling in C. elegans vulval development. Mol. Cell. 7, 461-473 (2001)

23. Nevins, J. R. The Rb/E2F pathway and cancer. Hum. Mol. Genet. 10, 699-703 (2001).

24. Leone, G. et al. Identification of a novel E2F3 product suggests a mechanism for determining specificity of repression by Rb proteins. Mol. Cell. Biol. 20, 3626-3632 (2000)

25. Cooper, C. S. et al. Nuclear overexpression of the E2F3 transcription factor in human lung cancer. Lung Cancer 54, 155-162 (2006).

26. De Meyer, T. et al. E2Fs mediate a fundamental cell-cycle deregulation in highgrade serous ovarian carcinomas. J. Pathol. 217, 14-20 (2009).

27. Foster, C. S. et al. Transcription factor E2F3 overexpressed in prostate cancer independently predicts clinical outcome. Oncogene 23, 5871-5879 (2004).

28. Ziebold, U., Lee, E. Y., Bronson, R. T. \& Lees, J. A. E2F3 loss has opposing effects on different pRB-deficient tumors, resulting in suppression of pituitary tumors but metastasis of medullary thyroid carcinomas. Mol. Cell. Biol. 23, 6542-6552 (2003).

29. Trikha, P. et al. E2f3 in tumor macrophages promotes lung metastasis Oncogene 35, 3636-3646 (2016).

30. Tsuruta, T. et al. miR-152 is a tumor suppressor microRNA that is silenced by DNA hypermethylation in endometrial cancer. Cancer Res. 71, 6450-6462 (2011).

31. Tang, Z. et al. GEPIA: a web server for cancer and normal gene expression profiling and interactive analyses. Nucleic Acids Res. 45, W98-W102 (2017).

32. Vasaikar, S. V., Straub, P., Wang, J. \& Zhang, B. LinkedOmics: analyzing multiomics data within and across 32 cancer types. Nucleic Acids Res. 46, D956-D963 (2018).

33. Lu, Y. \& Li, W. Functional characterization of E2F3b in human HepG2 liver cancer cell line. J. Cell. Biochem. 119, 3429-3439 (2017).

34. Al Ahmed, H. A. \& Nada, O. E2F3 transcription factor: a promising biomarker in lung cancer. Cancer Biomark. 19, 21-26 (2017).

35. Gao, Y. et al. MiRNAs and E2F3: a complex network of reciprocal regulations in human cancers. Oncotarget 8, 60624-60639 (2017).

36. American Cancer Society. Cancer Facts and Figures. https://www.cancer.org/ content/dam/cancer-org/research/cancer-facts-and-statistics/annual-cancerfacts-and-figures/2017/cancer-facts-and-figures-2017.pdf. Accessed 11 July 2017.

37. National Cancer Institute. Drugs Approved for Endometrial Cancer. https:// www.cancer.gov/about-cancer/treatment/drugs/endometrial. Accessed 11 July 2017.

38. Elstrom, R. L. et al. Akt stimulates aerobic glycolysis in cancer cells. Cancer Res. 64, 3892-3899 (2004)

39. Garg, S. K., Maurer, H., Reed, K. \& Selagamsetty, R. Diabetes and cancer: two diseases with obesity as a common risk factor. Diabetes Obes. Metab. 16, 97-110 (2014).

40. Eritja, N. et al. Endometrial carcinoma: specific targeted pathways. Adv. Exp. Med. Biol. 943, 149-207 (2017).

41. Tsuda, H., Yamamoto, K., Inoue, T., Uchiyama, I. \& Umesaki, N. The role of p16cyclin $d / C D K-p R b$ pathway in the tumorigenesis of endometrioid-type endometrial carcinoma. Br. J. Cancer 82, 675-682 (2000).

42. Ikeda, Y. et al. Prognostic importance of CDK4/6-specific activity as a predictive marker for recurrence in patients with endometrial cancer, with or without adjuvant chemotherapy. Br. J. Cancer 113, 1477-1483 (2015).

43. Westphalen, S. et al. Receptor mediated antiproliferative effects of the cytotoxic LHRH agonist AN-152 in human ovarian and endometrial cancer cell lines. Int. J. Oncol. 17, 1063-1069 (2000).

44. Shah, N. \& Sukumar, S. The Hox genes and their roles in oncogenesis. Nat. Rev. Cancer 10, 361-371 (2010)

45. Zhussupova, A. et al. An E2F1-HOXB9 transcriptional circuit is associated with breast cancer progression. PLoS One 9, e105285 (2014).

46. Kim, J. H. et al. Silencing of homeobox B9 is associated with down-regulation of CD56 and extrathyroidal extension of tumor in papillary thyroid carcinoma. Hum. Pathol. 43, 1221-1228 (2012).

47. Xue, M., Zhu, F. Y., Chen, L. \& Wang, K. HoxB9 promotes the migration and invasion via TGF-beta1/Smad2/Slug signaling pathway in oral squamous cell carcinoma. Am. J. Transl. Res 9, 1151-1161 (2017).

48. Wan, J. et al. PCAF-primed EZH2 acetylation regulates its stability and promotes lung adenocarcinoma progression. Nucleic Acids Res. 43, 3591-3604 (2015). 